A N N A L E S Annales de Bretagne et des Pays de l'Ouest

Anjou. Maine. Poitou-Charente. Touraine

$117-3 \mid 2010$

Usages et représentations du temps dans les sociétés littorales

\title{
Le temps des crises dans les sociétés littorales $\left(\mathrm{XVI}^{\mathrm{e}}\right.$ -XIX ${ }^{\mathrm{e}}$ siècle)
}

Gérard Le Bouëdec

\section{Q OpenEdition}

Journals

Édition électronique

URL : http://journals.openedition.org/abpo/1929

DOI : $10.4000 / a b p o .1929$

ISBN : 978-2-7535-1519-2

ISSN : 2108-6443

Éditeur

Presses universitaires de Rennes

Édition imprimée

Date de publication : 20 octobre 2010

Pagination : 13-24

ISBN : 978-2-7535-1276-4

ISSN : 0399-0826

Référence électronique

Gérard Le Bouëdec, "Le temps des crises dans les sociétés littorales (XVIe-XIXe siècle) 》, Annales de Bretagne et des Pays de l'Ouest [En ligne], 117-3 | 2010, mis en ligne le 02 mai 2011, consulté le 19 avril 2019. URL : http://journals.openedition.org/abpo/1929; DOI : 10.4000/abpo.1929 


\title{
Le temps des crises dans les sociétés littorales ( $\mathrm{XV} \mathrm{I}^{\mathrm{e}}-\mathrm{XIX} \mathrm{X}^{\mathrm{e}}$ siècle)
}

\author{
Gérard LE BOUËDEC \\ Professeur d'histoire moderne \\ CERHIO, UMR 6258 - université de Bretagne-Sud
}

Entre une histoire économique qui analyse concrètement les faits et une économie politique qui s'intéresse à l'analyse abstraite des mécanismes généraux, l'historien est souvent critiqué par les économistes qui lui reprochent une insuffisance d'analyse macro-économique et une tendance à se livrer à une description qui relève de la chronique événementielle. Les historiens qui travaillent sur le temps long sont pris entre les schémas d'une histoire des crises d'Ancien Régime qui reste encore largement marquée par les acquis labroussiens de l'histoire de la conjoncture et les schémas de la Crise, qui s'inscrit dans le mouvement cyclique des économies industrielles et des structures capitalistes ${ }^{1}$. Olivier Pétré-Grenouilleau a livré une analyse des grands cycles de l'économie maritime française du xvII au XIX $X^{e}$ siècle en identifiant les phases d'émergence, de plein exercice et de restructurations ${ }^{2}$.

Cet article s'inscrit dans une démarche méthodologique pour montrer l'intérêt de l'étude des ruptures dans les sociétés littorales, le terme de rupture paraissant plus adapté que celui de crise. En réalité nous avons un problème de vocabulaire. Le plus souvent la rupture se révèle dans nos sources comme une phase difficile vécue par un groupe social ou une communauté plus large qui exprime alors ses inquiétudes voire ses colères. II est possible de trouver les allégations dans notre documentation à travers des mémoires, des rapports, des articles de presse qui exposent ce qu'Alain

1. LABRousse, Ernest, La Crise de l'économie française à la fin de l'Ancien Régime et au début de la Révolution, 1943 ( $2^{\mathrm{e}}$ édition) Paris, Presses universitaires de France, 1990, 664 p.). Voir également BRAudel, Fernand, LABRousSE, Ernest, Histoire économique et sociale de la France, Paris, Presses universitaires de France, 1970. Sur les productions historiques de l'école des Annales, voir Bourdé, Guy, MARTIN, Hervé, Les Écoles historiques, Paris, Seuil, 1983, chapitre 9, p. 215-243.

2. Pétré-Grenoullleau, Olivier, Les Négoces maritimes français ( $x V I I^{e}-x x^{e}$ siècles), Paris, Belin, 1997, 255 p. 
Cabantous appelle des faits maritimes ${ }^{3}$ et qui sont de nature très différente. C'est à partir de ce niveau que se construit le temps de l'historien. En effet, ces faits maritimes doivent être mis en perspective. Ils s'inscrivent dans la trajectoire particulière de chaque bourg ou ville du littoral qui s'éclaire dans le cadre des trajectoires générales des différents modèles de développement qui affectent les économies et les sociétés littorales. Ainsi, il s'agit d'un plaidoyer pour l'étude des temps à différentes échelles des communautés littorales et portuaires.

\section{De la rupture vécue aux faits allégués}

Les sources sont riches de l'expression des plaintes, des drames humains et des manifestations de ces déséquilibres qui menacent la survie d'une activité, d'un groupe socioprofessionnel, d'une communauté dans son ensemble. C'est le malvécu qui nous sert d'indicateur. Les épidémies de peste comme celle de Marseille en 1720 ou de typhus à Brest lors de la guerre de Sept ans, l'effet meurtrier et destructeur de Guy Eder de la Fontenelle et de ses bandes de pillards sur l'avenir de Penmarch pendant les guerres de la Ligue en Bretagne à la fin du $\mathrm{xvl}^{\mathrm{e}}$ siècle ${ }^{4}$, la répression contre le port de La Rochelle en 1627-1628 orchestrée par Richelieu ${ }^{5}$, la multiplication des abandons - placements d'enfants mais aussi des naissances illégitimes à Lorient pendant la guerre de Sept $\mathrm{Ans}^{6}{ }^{-}$, les bombardements et les débarquements qui se multiplient sur le littoral français pendant la seconde guerre de Cent Ans, à l'exemple de ce qui se passe à Belle-île entre 1761 et 1763, ou à Dunkerque en 1713 ou $1763^{7}$, mais qui sont incomparables avec les effets destructeurs de la seconde guerre mondiale qui vont frapper en particulier tous les ports dotés par les Allemands de base de sous-marins, s'inscrivent en lettres dramatiques dans I'histoire des communautés littorales. Le tribut payé par les gens de mer au système des classes lors des guerres est de nature à déstabiliser toute une activité et à hypothéquer l'avenir de certaines paroisses littorales ${ }^{8}$. Ainsi, la mort,

3. CABAntous, Alain, "L'Histoire maritime : objet de recherche ou leurre historiographique? » dans Villain-Gandossı, Christiane, Rieth, Eric, Pour une Histoire du "Fait maritime ", sources et champ de recherche, Paris, Édition du Comité des travaux historiques, 2001, p. 33-43.

4. Lespagnol, André, Tanguy, Jean., "Penmarch, port européen aux $x V l^{\mathrm{e}}-\mathrm{xVII}{ }^{\mathrm{e}}$ siècles. Réflexion sur un destin problématique, le pays bigouden à la croisée des chemins », Revue du Cap Caval, $\mathrm{n}^{\circ} 17,1993$.

5. CRETE, Liliane, La Vie quotidienne à La Rochelle au temps du grand siège : 1627-1628, Paris, Hachette, 1987, 333 p.

6. LE BOUËDEC, Gérard, "Les enfants de I'Hôtel-Dieu de Lorient : illégitimité et misère pendant la guerre de Sept Ans », Cahier de I'Iroise, octobre-décembre 1986, p. 181-187.

7. Verge-Franceschi, Michel, La Marine française au XVIII ${ }^{\mathrm{e}}$ siècle, Paris, Sedes, 1996, p. $217-$ 257. LE BOUËDEC, Gérard, Activités maritimes et société littorales de l'Europe atlantique (1690-1790), Paris, A. Colin, 1997, p. 182-186.

8. AcerRA, Martine, Zysberg, André, L'Essor des Marines européennes, Paris, Sedes, 1997, p. $258-260$. 
la détresse des familles, la destruction des outils de travail, la fuite constituent les expressions de l'ensemble de la communauté.

Les vols, les grèves, les manifestations révèlent de tout temps les craquements des activités productrices. Des bandes d'ouvriers qui se livrent à des vols parcourent les campagnes de la périphérie lorientaise, quand le port perd sa compagnie des Indes dans les années $1769-1770^{9}$. Les milieux négociants des grands ports français, à l'instar de ceux de Nantes, se plaignent de voir les étrangers, notamment hollandais, ôter "tout le trafic, commissions et correspondances " aux acteurs locaux. Ces plaintes se retrouvent dans "le commerce honorable » de Jean Eon en 1656 à Nantes, notamment dans le chapitre " calcul exact de tout le grand négoce, et du profit que font les étrangers au préjudice des Français ${ }^{10} »$. Les registres des faillites des consulats et des tribunaux de commerce nous livrent la chronique des revers des élites du commerce et de l'industrie ${ }^{11}$ sans qu'il soit possible d'en tirer des conclusions définitives. La chute des plans de charge dans les arsenaux est à l'origine de tensions dès l'Ancien Régime. Les années 1890-1914 correspondent à une vague de grèves dures parmi les dockers et les ouvriers des industries portuaires ${ }^{12}$. La disparition de la ressource, au début du XvIII ${ }^{\text {e }}$ siècle, dans les années 1880 , secoue les communautés de pêcheurs. Les marins-pêcheurs et leurs familles, privés d'emplois et de revenus dans les années 1880-1920, du fait de la raréfaction des bancs et des fermetures des conserveries de sardines, expriment leur détresse à travers grèves et manifestations parfois violentes ${ }^{13}$, des choix particuliers comme l'engagement de pêcheurs concarnois dans les mines de Courrières ${ }^{14}$. Les réactions des pêcheurs contre les chaluts, les conflits d'usages sur les plages entre d'une part les pêcheurs qui étendent leurs filets, les agriculteurs qui y puisent du sable, les goémoniers qui y font sécher le produit de leur collecte et d'autre part les baigneurs qui ne sont pas encore des touristes, qui colonisent l'estran avec leurs cabines de bain ou leurs bath-machines, expriment aussi la crainte d'une dilution d'un modèle social et culturel devant ces intrusions de nouvelles techniques ou de communautés exogènes ${ }^{15}$.

9. LE BOUËDEC, Gérard, Le port et l'arsenal de Lorient, de la compagnie des Indes à la Marine cuirassée, une reconversion réussie (XVIII ${ }^{\mathrm{e}} \mathrm{xIX} \mathrm{X}^{\mathrm{e}}$ siècle), Paris, Librairie de l'Inde, 1994, p. 221-224.

10. Eon, Jean, Le Commerce honorable, Nantes, 1656.

11. Série $B$, juridictions extraordinaires des archives départementales.

12. Domenichino, Jean, Guillon, Jean-Marie, Mencherinı, Robert (dir.), Dockers, de la Méditerranée à la mer du Nord. Des quais et des hommes dans I'histoire, Aix-en-Provence, 1999, 238 p.

13. Dubols, Xavier, La Révolution sardinière, pêcheurs et conserveurs en Bretagne sud au XIX ${ }^{e}$ siècle, Rennes, presses universitaires de Rennes, 2004, p. 199-312. LE BouLANGER, JeanMichel, Douarnenez, de 1800 à nos jours. Essai de géographie historique sur l'identité d'une ville, Rennes, PUR, 2000, p. 173-238.

14. Gueguen, Michel, Lemaître, Louis-Pierre, Matelots de Concarneau (1800-1914), Concarneau, 1979, p. 322.

15. VINCENT, Johan, L'Intrusion balnéaire, les populations littorales bretonnes et vendéennes face au tourisme (1800-1945), Rennes, PUR, 2007, p.163-179. 
Ce n'est qu'une évocation des différentes formes d'expression des ruptures vécues. En effet nous sommes confrontés à différents problèmes.

Si les grandes inondations de 1548 et le raz de marée de 1598 qui affectent la baie de Bourgneuf, les tempêtes qui menacent Saint-Jean-de-Luz comme celle de 1749 qui contraint à abandonner 120 maisons, sont des faits durement subis, comment apprécier le ressenti des processus lents, qui s'étalent sur plusieurs générations de communautés littorales, que sont les envasements qui vont condamner Brouage, les ports de la Seudre et ceux de la baie de Bourgneuf entre le $x \mathrm{VI}^{\mathrm{e}}$ et le $\mathrm{xvII^{ \textrm {e } }}$ siècle ${ }^{16}$ ?

De la même manière un ensemble de ruptures nous échappe car l'exercice des activités s'inscrit dans une gestion pluriactive des territoires littoraux. Cette pluriactivité, notamment dans les bourgs ruraux, permet d'absorber les à-coups ou les ratés de la conjoncture maritime. Ce n'est que lorsque se combinent une mauvaise récolte de grains et une mauvaise campagne de pêche comme en 1741 à Belle-Île que les sources sont plus loquaces. Aussi, sommes-nous souvent mieux renseignés sur les crises et les ruptures dans les grandes villes-ports de commerce et de la marine militaire que dans les petits ports de cabotage et de pêche. Ce n'est qu'avec l'avènement de l'ère industrielle qui s'accompagne d'une spécialisation et d'une professionnalisation dans les métiers de la pêche, de la navigation, de l'industrie portuaire que l'expression de la crise apparaît plus forte d'autant qu'elle s'étale dans la presse avant d'être relayée dans les nouveaux médias du son et de l'image.

Nous sommes bien face à un problème de sources. Un premier ensemble est produit par des observateurs extérieurs : officiers, commissaires royaux, intendants, préfets, médecins, missionnaires, ingénieurs, voyageurs écrivains ou artistes, journalistes ${ }^{17}$. L'étatisation du littoral, à partir de Colbert, qui se traduit par la mise en place d'un maillage serré de juridictions, multiplie les institutions productrices d'archives sur les populations littorales désormais très largement sous le contrôle des agents de l'État. Les sources de ceux qui vivent au cœur du milieu littoral et maritime sont aussi plurielles, mais moins nombreuses. Avant les cahiers de doléances, les expressions directes des communautés littorales et rurales sont rares ${ }^{18}$. Plus fréquents sont les

16. Ports maritimes de France, tomes V, VI, VII, Imprimerie nationale, 1883 : Voir les notices historiques des ports réalisées par les ingénieurs des Ponts et chaussées.

17. CABANTOUS, Alain, "La mémoire du voyageur: sociétés et espaces littoraux des XVII ${ }^{\mathrm{e}}$ XIX ${ }^{\mathrm{e}}$ siècles ", table ronde organisée à I'Université de Bretagne-Sud en janvier 1995. Pour approfondir voir CABANTOUS Alain et alii, Les Français, la terre et la mer, Paris, Fayard, 2005, p. 359-368.

18. VARY, Morgane., Intégration sociale des populations marginales sur le littoral breton au XVIII ${ }^{e}$ siècle, thèse d'histoire inédite, Université de Bretagne Sud, 2007. Morgane Vary nous fait découvrir une nouvelle strate de ces sociétés littorales, plus inconnue encore que la précédente, celle des populations flottantes et migrantes qui constituent d'une certaine façon les bas-fonds de cette société littorale. Or ces marginaux, dont elle reconstitue les trajectoires de déchéance qui les avaient classés dans la catégorie des exclus, révèlent au gré des archives judiciaires, du dépôt de mendicité ou du bagne, un autre visage pour le peu que l'on veuille s'intéresser aux tranches de vie qui s'insèrent entre leurs arres- 
mémoires adressés par le monde du négoce, à travers les consulats et les chambres de commerce, ou par les municipalités, mais qui sont aux mains de ces mêmes élites, aux autorités de l'État. Dans certaines circonstances particulières des curés ou des agents de l'état qui vivent au cœur du tissu social local peuvent donner des avis et des opinions qui ne figurent pas dans les dossiers institutionnels. L'ensemble de cette documentation exige une grande prudence et une analyse critique rigoureuse pour comprendre l'objectif des auteurs et ainsi décoder le discours qui sous-tend les observations. Ainsi de nombreux mémoires de négociants et de municipalités dressent des tableaux pessimistes. C'est la loi du genre quand il s'agit de plaider des aides, des privilèges auprès d'autorités supérieures. II en est ainsi des mémoires sur l'invasion étrangère des ports au XvII ${ }^{\mathrm{e}}$ siècle. Damien Mouries ${ }^{19}$, à partir d'un corpus de mémoires de marchands, d'agents de la royauté et de publicistes a montré la généalogie de ces écrits qui présentent de nombreuses filiations. Le parfait négociant de Jacques Savary ${ }^{20}$, comme le Commerce honorable de Jean Eon se sont nourris d'autres mémoires. Surtout, ils sont écrits pour susciter une autre politique économique et de ce fait font la démonstration de la dégradation du commerce français en dénonçant les coupables : les négociants étrangers. Les mémoires des chambres de commerce sur un éventuel retour du négoce français dans le commerce du nord au XVIII ${ }^{\mathrm{e}}$ siècle soulignent toujours le complexe d'infériorité des Français pour exiger des aides de l'État ${ }^{21}$.

Au XIX siècle et a fortiori ensuite, la production de sources va croître et se diversifier du fait de la structuration progressive de contre-pouvoirs. Les municipalités, les chambres de commerce s'érigent en véritables lobbies pour défendre leurs activités, et au niveau national, les armateurs et les constructeurs de navires savent se faire entendre d'un État en décrochant subventions et commandes ${ }^{22}$. La lecture des débats parlementaires est à ce titre très édifiante. L'argumentaire social n'est pas du tout absent même s'il est parfois un prétexte. Dans les villes-port-arsenal, les élus ne manquent de rappeler au ministère la dimension sociale d'une politique maritime militaire ${ }^{23}$. Les marins, les dockers, les ouvriers des arsenaux et

tations. Elle arrive alors à démontrer en s'appropriant et en renouvelant le concept de pluriactivité, qui aujourd'hui n'est plus contesté, que ces hommes et ses femmes ne sont pas, pour paraphraser le mot d'Arlette Farge, si marginaux que cela, et que la dialectique exclusion/intégration, problématique intemporelle, mérite d'être approfondie.

19. MouriES, Damien, Les Milieux négociants français et le commerce intra-européen au $\mathrm{XVII}^{\mathrm{e}}$ siècle, mémoire de DEA, Université de Bretagne Sud, 2004.

20. SAVARY, Jacques, Le parfait négociant, Paris, $1^{\text {re }}$ édition $1675,324 \mathrm{p}$.

21. POURCHASSE, Pierrick, Le Commerce du Nord, les échanges commerciaux entre la France et l'Europe septentrional au XVIII' siècle, Rennes, PUR, 2006, p. 217-262.

22. BoRDE, Christian, "La création du Comité Central des Armateurs de France (13 janvier 1903) : enjeux internationaux et nationaux du lobbying maritime français (18911906) », Revue d'Histoire maritime, $n^{\circ}$ 5, p. 221-237.

23. LE BOuËDEC, Gérard, "L'État et les ports bretons de Brest et de Lorient de Colbert au $x x^{e}$ siècle » dans Nicolas, Gilbert, La Construction de I'Identité régionale : les exemples de la Saxe et de la Bretagne, $x V I I{ }^{e}-x x^{e}$ siècle, Rennes, PUR, 2001, p. 43-54. 
des industries agro-alimentaires, les pêcheurs se syndicalisent même si la fracture entre les syndicats corporatifs des pêcheurs et les marins-ouvriersdockers qui adhèrent à la CGT d'une part, et l'atomisation progressive du syndicalisme maritime d'autre part lui ôtent un véritable poids ${ }^{24}$. Mais ce n'est pas un hasard si les années 1880-1914 constituent une grande période de contestations.

Si les sources sont majoritairement institutionnelles sous l'Ancien Régime, elles apparaissent davantage nourries par les acteurs professionnels, locaux et nationaux, ensuite. Le XIXe siècle est celui de l'intégration des mondes littoraux dans la communauté nationale, c'est la rencontre entre ceux du rivage et ceux de l'intérieur. Par la suite, les littérateurs et les artistes, les photographes et les journalistes vont devenir de grands producteurs de sources. Le reportage devient une source à manier avec soin.

Trajectoire individuelle et trajectoire collective

L'ensemble des informations doit être hiérarchisé. La grille de lecture est d'abord constituée par les trajectoires générales de développement de l'ensemble des littoraux et surtout par les corrections de trajectoires qui constituent les ruptures majeures ${ }^{25}$.

À la fin du Moyen Âge, le rapport à la mer se construit à travers les pêches côtières et le cabotage et la poussière portuaire va s'appuyer sur ces activités jusqu'au milieu du XIX ${ }^{\mathrm{e}}$ siècle. Pourtant, dès la fin du $\mathrm{Xv}^{\mathrm{e}}$ siècle la pêche morutière devient transatlantique, mobilisant de nombreux ports avant un reflux sur quelques ports majeurs. Le milieu du $\mathrm{xvII}^{\mathrm{e}}$ siècle correspond à la première mondialisation des échanges avec le développement des systèmes coloniaux tandis que s'observe une accélération du désenclavement européen. Le cabotage européen qui s'appuyait sur des produits vivriers, la proto-industrie textile, et quelques matières premières, est gonflé par les navals stores (bois, fer, chanvre, goudron) d'une part et les réexportations des produits coloniaux, d'autre part. Mais l'armement français est alors écarté par les flottes hollandaises, anglaises et scandinaves. Seuls les ports qui ont fait le choix du commerce colonial se hissent au rang de grands ports. Mais cette mondialisation des échanges, c'est la guerre. Les littoraux deviennent les nouveaux fronts des conflits internationaux et les grandes puissances vont construire des flottes de guerre spécialisées et implanter une série de ports-arsenaux. Le $\mathrm{XIX}^{\mathrm{e}}$ siècle introduit les littoraux dans la modernité. Le commerce maritime va refléter l'entrée dans l'ère industrielle. Les échanges sont dominés par les matières premières indus-

24. VIAUD, Ronan, Le Syndicalisme maritime français. Les organisations, les hommes, les luttes (1890-1950), Rennes, PUR, 2005, 278 p.

25. Cabantous, Alain, Les Citoyens du large, les identités maritimes en France (xvII $\mathrm{e}_{-}$ XIX siècles), Paris, Aubier-Montaigne, 1995, p. 161-167. Coulıou, Jean-René, Le BouËDEC, Gérard, Les ports du Ponant : I'Atlantique de Brest à Bayonne, Éditions Palantines, 2004, voir le chapitre, «Les destins portuaires », p. 236-259. 
trielles. Le centre de gravité de l'Europe se déplace vers le nord-ouest. La révolution des transports maritimes et terrestres transforme le port en lieu de transbordement et en centre industriel. Cette reconfiguration des échanges et des transports signifie le déclassement de nombreux ports. Les activités halieutiques sont quant à elles en plein développement. La grande pêche Terre-neuvière retrouve son dynamisme tandis que s'ouvre plus largement un second front morutier en Islande mais dont les activités s'essoufflent au début $d u x^{e}$ siècle. Les pêches sardinières connaissent une forte croissance en liaison avec l'industrialisation du traitement du poisson dans les conserveries, mais les crises sardinières fragilisent les ports de la Bretagne occidentale à la Vendée. La mise au point du chalutage à vapeur fait entrer la pêche au poisson frais dans l'ère de la pêche hauturière. La Marine militaire connaît, elle aussi, sa révolution technologique, et les arsenaux deviennent de grands conglomérats industriels dont les plans de charge oscillent au gré des programmes militaires et des budgets. II ne faut pas omettre que l'activité balnéaire et de plaisance va amorcer à partir des années 1840-1850 un développement qui va toucher l'ensemble des littoraux. Cette lecture macroscopique donne quelques repères qui éclairent les trajectoires particulières des ports.

Mais il faut compléter la grille de lecture en se référant aux évolutions de chaque activité, de chaque filière. Les pêches morutières et sardinières notamment ont leur propre trajectoire. Les ports salicoles sont particulièrement touchés par des décisions essentielles qui touchent le commerce du sel dans les années 1840-1848. Les ports militaires présentent des profils à pulsions brutales, la guerre impulsant une croissance forte des activités suivie d'un ralentissement tout aussi brutal ${ }^{26}$. La guerre est destructrice mais aussi créatrice notamment à travers la course. Le projet de réduire le dispositif militaire à trois arsenaux avec la construction de celui de Cherbourg va peser sur l'avenir de Lorient et surtout de Rochefort.

C'est par rapport à cette double grille de lecture qu'il est alors possible d'examiner et d'éclairer les faits de rupture individuels en considérant qu'à chaque étape se produit une sélection avec promotion et déclassement. Que constate-t-on? Sur le temps long, les ports ne meurent jamais. En revanche, ils connaissent des reclassements souvent radicaux. Les ports de cabotage, qui constituent le substrat de la carte portuaire française, après avoir connu des difficultés lors de la reconfiguration des transports et des échanges dans les années 1850-187027, se replient sur des trafics de proximité, qui repoussent l'échéance de leur endormissement jusqu'à la première guerre mondiale. Ils vont souvent retrouver une autre vie avec

26. Jean Meyer qualifie ces villes ports-arsenaux de "villes quasars ». Voir BÉRENGER, Jean, MEYER, Jean, La France dans le monde au XVIII siècle, Paris, Sedes, 1993, p. 305.

27. Le Gouguec, Anne-Françoise, Les mutations du cabotage au XVIII ${ }^{\mathrm{e}}$ siècle et au XIX $\mathrm{X}^{\mathrm{e}}$ siècle, depuis les rôles de bord des maîtres au cabotage des quartiers de Vannes et d'Auray, mémoire de DEA, Université de Bretagne Sud, 2001. LE BouËDEC, Gérard, " Les enjeux du cabotage européen (XIVe-XIXe siècle) », dans BuCHET, Christian, MEYER, Jean, Poussou, Jean-Pierre, La Puissance maritime, Presses universitaires de la Sorbonne, p. 377-394. 
les activités de plaisance. Le port de Yeu, port de cabotage de service de Bordeaux et de Nantes, notamment aux $x \mathrm{VII}^{\mathrm{e}}$ et $\mathrm{xVII{ } ^ { \mathrm { e } }}$ siècles, va s'engager dans la pêche notamment thonière au XIX ${ }^{\mathrm{e}}$ siècle ${ }^{28}$. Les grands ports français que nous connaissons, comme Le Havre, Nantes, Bordeaux, qui s'étaient développés sur le cabotage et la pêche morutière vont engager une reconversion radicale fin $\mathrm{XVII}^{\mathrm{e}}$, début $\mathrm{XVIII}{ }^{\mathrm{e}}$ siècles, vers le commerce antillais et connaître alors un décollage. Ces ports restent au XIX siècles des grands ports français, même s'ils sont décrochés par les grands ports du nord de l'Europe et qu'il faille inventer Saint-Nazaire pour remplacer Paimbœuf comme avant-port de Nantes. Des ports polyvalents vont se replier sur la pêche comme Saint-Malo et Dieppe, des ports de grande pêche comme Saint-Jean-de-Luz et Les Sables-d'Olonne vont se recentrer sur les pêches côtières. Des ports de pêche sardiniers vont s'orienter vers les pêches thonières (Groix, Étel, Concarneau) et langoustières (Douarnenez, Camaret) ${ }^{29}$. Le port harenguier de Boulogne connaît un grand développement avec le chalutage à vapeur du poisson frais à partir de la fin du XIXe siècle Le monolithisme des ports militaires n'est guère remis en cause depuis Louis XIV. En trois siècles, L'arsenal mixte de Lorient a toujours été confirmé alors qu'il était le plus menacé ce qui explique que la Marine a accepté sa diversification vers le commerce du charbon et le chalutage à vapeur. Cherbourg a connu un développement très rapide à partir du Second Empire. Seul Rochefort est fermé en 1927.

Au final, une conclusion, à ce stade de l'analyse, s'impose. Les ruptures majeures sont des corrections de trajectoires, des ajustements, des reconversions, qui s'inscrivent dans un temps qui n'est pas le temps court, mais qui s'étalent sur une ou deux générations des acteurs voire davantage. Olivier Pétré-Grenouilleau identifie trois intercycles majeurs où cohabitent le modèle finissant et le modèle émergent, le XVII ${ }^{\mathrm{e}}$ siècle, 1760-1840, 1880-1890. Or les sources bruissent davantage d'événements ponctuels insupportables quand ils sont répétitifs. Ils révèlent soit des accidents de parcours sans lendemain, soit des à-coups du modèle en place, soit encore constituent des signaux d'alerte d'une rupture plus radicale, mais qui ne sont pas toujours perceptibles par ceux qui les vivent. Alain Cabantous intègre les deux dimensions du temps dans un sous-chapitre intitulé " les menaces » dans une partie consacrée aux stratégies de l'identité dans son ouvrage « Les citoyens du large ${ }^{30} »$.

28. Il suffit de lire successivement l'ouvrage de HENRY, Jean-François, Des marins au siècle du Roi Soleil, Salmon, 1982 et Brigand, Louis, Les îles du Ponant, Éditions Palantines, 2002, p. 220-221.

29. LE BOUËDEC, Gérard, "Les reconversions des pêches sardinières sur le littoral de Bretagne occidentale et méridionale (1880-1930) : le rapport à l'innovation », dans DANIC, Guy, Geistdoefer, Aliette, Le BouËDec, Gérard, THeret, François, Les Mutations techniques des pêches maritimes: Agir ou subir, évolution des systèmes techniques et sociaux, IFREMER 2001, p. 31-40.

30. CABAntous, Alain, Les Citoyens du large, op. cit., p. 161-176. 
Le temps de l'historien, l'étude des ruptures

Alain Cabantous considère que la seule manière de faire de l'histoire maritime et littorale est de prendre pour objet d'étude des faits qui permettent de mesurer le rapport à la mer et au littoral « un événement répétitif, un comportement collectif, un produit échangé, une communauté urbaine ou rurale $^{31} »$. Marcel Mauss avait ouvert le chemin pour l'étude du fait social total $^{32}$. Si nous voulons sortir d'une approche trop événementielle qui fait la part trop belle aux événements courts et bruyants, il faut prendre pour objet d'étude ces ruptures, ces changements de trajectoires.

En creux, lors de l'étude de la déstructuration, se révèle le modèle finissant, le jeu des acteurs, les liens sociaux, l'articulation entre le local et les autres échelles, en positif la reconstruction, le tissage des liens entre des acteurs renouvelés ou pas du nouveau modèle dans toutes ses dimensions économiques, sociales, culturelles et politiques. L'expression du vécu ou du mal vécu s'éclaire alors dans ce cadre. Le terme de rupture est plus neutre que celui de crise qui est nécessairement connoté négativement. Or nous saisissons davantage les processus de déstructuration car destructeur et donc générateur de tensions. Il s'agit ici de comprendre un processus à deux volets, déconstruction et reconstruction, de mesurer le rapport au changement subi ou choisi, de mesurer le rapport à l'innovation des acteurs, d'étudier la dynamique sociale ${ }^{33}$.

Les travaux sur les nouvelles trajectoires des activités sont fréquents. Ainsi nous disposons dans Les négoces maritimes français $\mathrm{XVII}{ }^{\mathrm{e}}-\mathrm{xx} \mathrm{e}^{\mathrm{e}}$ siècles d'Olivier Pétré-Grenouilleau ${ }^{34} \mathrm{~d}^{\prime}$ une analyse des trajectoires du grand capitalisme maritime français. Néanmoins, il manque particulièrement des études sur les modalités sociales, culturelles et politiques de ces corrections de trajectoires qui nécessitent des travaux en zoom sur les communautés littorales et portuaires et des remises en perspective dans des grandes synthèses des productions publiées ou grises isolées. Globalement, il manque encore des synthèses sur les grandes ruptures de la deuxième moitié $d u x_{V I I}{ }^{e}$ siècle, sur les reclassements de la première moitié du XIXe siècle, qui s'expliquent en partie par le manque d'études économiques et sociales des communautés durant la période de la Révolution et de l'Empire ${ }^{35}$. Nous n'avons que des premiers résultats sur le devenir

31. CABANTOUS, Alain, "I'histoire maritime : objet de recherche ou leurre historiographique? », art. cit., p. 33-43.

32. Mauss, Marcel, Sociologie et anthropologie, Paris, Presses universitaires de France, $1950,389 \mathrm{p}$.

33. LE BOUËDEC, Gérard, «Les reconversions des pêches sardinières sur le littoral de Bretagne occidentale et méridionale (1880-1930) : le rapport à l'innovation », dans : DANIC, Guy., Geistdoefer, Aliette, Le Bouëdec, Gérard, Theret, François, op. cit.

34. Pétré-Grenouilleau, Olivier, Les Négoces maritimes français, op. cit.

35. AudRAN, Karine, Les Négoces portuaires bretons sous la Révolution et l'Empire, bilan et stratégies : Saint-Malo, Morlaix, Brest, Lorient et Nantes, 1789-1815, thèse d'Histoire inédite soutenue à l'Université de Bretagne Sud., 2007. L'histoire économique est restée le parent pauvre de l'historiographie révolutionnaire. J.-C. Perrot soulignait en 1970 que c'était une 
des pôles caboteurs décrochés dans les années 1850-1870 en Bretagne, nous savons que le balnéaire n'a jamais été perçu comme une solution alternative aux difficultés des activités traditionnelles, que la Marine militaire n'est pas non plus vue comme une solution de reconversion pour des communautés halieutiques pourtant fragilisées du moins au $\mathrm{xIX}^{\mathrm{e}}$ siècle ${ }^{36}$. II faut s'emparer de la problématique de l'innovation dans les communautés de taille modeste, qu'elle soit technique, financière et socio-culturelle, pour arrêter d'opposer la modernité absolue des grands ports et l'archaïsme des communautés portuaires modestes ${ }^{37}$.

Le temps des crises et le temps des ruptures recouvrent des réalités différentes. La crise correspond à un temps social court où s'expriment les difficultés de tout ou partie des acteurs. Les accidents naturels, les revers de la conjoncture, les mauvaises campagnes de pêche, l'interruption des activités pendant les guerres fragilisent les communautés. Leur étude est alors un moyen de comprendre le fonctionnement du modèle. La crise est un miroir pour découvrir l'organisation et le fonctionnement de la communauté au cœur d'un modèle.

Le temps des ruptures est beaucoup plus complexe et souvent long car coexistent les difficultés d'un modèle avec la construction d'un nouveau modèle. À Lorient la reconversion du modèle marchand vers un modèle militaire s'étale de 1770 à $1830^{38}$. Les ports morutiers du complexe portuaire nantais comme Les Sables-d'Olonne, sont incapables de trouver leur place dans le système antillais que construisent les Nantais. La construction du modèle nantais est mieux connue que le déclin de la pêche morutière sablaise, mais les Sables ont alors fait d'autres choix ${ }^{39}$. Les efforts d'adaptation sont parfois vains. Les maîtres au cabotage du Golfe du Morbihan ont su mobiliser le micro-capitalisme local pour construire une flotte de service de gros chasse-marée, mais passées les années 1860, elle est totale-

zone d'affaiblissement historiographique. Lors du bicentenaire, Michel Vovelle confirmait la faiblesse de la contribution de I'histoire économique et il est vrai que l'histoire politique dominait la production historiographique. On ne sortait pas de la dialectique catastrophe/libération économique. On assiste à une revitalisation du champ économique depuis la fin des années 80 , le modèle de la crise et du déclin est remis en cause par Sylvia Marzagalli et Olivier Pétré-Grenouilleau qui défendent la thèse de l'adaptation, de l'attente et de la réorientation. Cette thèse s'inscrit dans ce courant. Les déficiences de I'Historiographie économique constituaient en outre un handicap pour l'histoire du commerce maritime, pour lequel l'entrée dans l'ère industrielle constitue une rupture plus pertinente. Néanmoins faute d'étude, l'enjambement de la période révolutionnaire et impériale plus ou moins déguisé était classique mais hypothéquait la pertinence de l'argumentation sur la reprise et le développement (ou leur décrochage) des ports et des échanges dans la première moitié $d u$ xIx ${ }^{\mathrm{e}}$ siècle. La thèse de Karine Audran répond avec des acquis essentiels.

36. VINCENT, Johan, L'Intrusion balnéaire, op. cit.

37. Voir notre chapitre "Entre traditions et innovations » dans CABANTOUs, Alain, LESPAGNOL, André, PÉRON, Françoise (dir.), Les Français, la terre et la mer, op. cit.

38. LE BOUËDEC, Gérard, Le Port et l'arsenal, op. cit.

39. Michon, Bernard, L'aire portuaire de Nantes aux XVII et $x{ }^{e} I I I^{e}$ siècles, thèse $d^{\prime} h i s t o i r e$ inédite soutenue à l'université de Nantes, 2005. 
ment incapable de résister à la nouvelle reconfiguration des échanges et du transport maritime ${ }^{40}$. Parallèlement le golfe du Morbihan s'est lancé dans l'ostréiculture ${ }^{41}$ puis le tourisme balnéaire. Le lien entre la disparition d'un système et l'émergence de nouvelles activités reste à établir.

Pour l'historien, c'est moins la réussite que le projet, la stratégie développée, la mobilisation des moyens, le jeu des acteurs qui comptent. II faut sans doute arrêter de se focaliser sur ce qu'on appelle les échecs qui supposent une échelle des valeurs. À l'époque de Colbert, la référence était les Provinces-Unies, au XIX siècle, l'Angleterre. Nous avons à l'esprit des normes, des modèles de référence. II faut s'en détacher pour comprendre les trajectoires particulières des communautés littorales.

La crise et la rupture de trajectoire constituent les deux temps qui marquent la vie des sociétés littorales. Le diagnostic de la crise permet d'ausculter dans toutes ses dimensions un système. Le champ de recherche de la rupture est beaucoup plus complexe. Ces périodes de l'entre-deux sont rarement étudiées faute sans doute de disposer des outils conceptuels adaptés à l'échelle des communautés littorales. Ainsi entre l'histoire des représentations et l'histoire des grands cycles économiques, entre microhistoire et histoire globale, il y a une place pour l'étude des communautés littorales et portuaires dans le vécu et la construction de leur trajectoire ${ }^{42}$.

40. BRIENT, Xavier, L'armement au cabotage en Bretagne Sud (1815-1848), Mémoire de DEA, Université de Bretagne Sud, 2003.

41. LE BiHAN, Janine, Ostréiculture et société ostréicole en Bretagne sud (1850-1986), thèse d'Histoire inédite, Université de Bretagne Sud, 2007.

42. Voir notre étude des communautés halieutiques de Bretagne Sud du XVIII au début du $x x^{e}$ siècle avec LE HUNSEC, Mathieu et MALONDA, Frédéric, "Les inscrits à la petite pêche et leur rapport au service de la Marine », Revue d'Histoire maritime, $n^{\circ} 7$, janvier 2008, p. 187-205. 
RÉSUMÉ

Cet article s'attache à montrer l'intérêt d'une analyse des crises et des ruptures qui, à l'époque moderne ou au XIX siècle, affectaient les sociétés littorales. Pour observer comment les populations des bourgs, des ports ou des villages de l'interface littorale percevaient et vivaient les crises économiques, mais aussi les guerres ou les accidents tels que les raz de marée, I'historien dispose de sources très variées dont on évoquera la nature. On verra que l'étude des trajectoires individuelles ou collectives, des ajustements et des conversions permet d'observer comment les groupes ou les différentes filières s'adaptaient aux bouleversements qui les affectaient.

\section{ABSTRACT}

This paper tries to show the great interest of studying the periods of crisis and abrupt changes that affected the seashore societies between the $16^{\text {th }}$ and the $19^{\text {th }}$ Centuries. How did people who lived in ports or in villages settled near the sea perceive economic crisis, wars, or accidents such as tidal waves? How did individuals, groups or professions adapt to disturbing events or long-term change? And what was the nature of this adaptation? The author also lists the type of sources that can be used to study those questions. 